\title{
Identification of a putative inhibitor of early pregnancy factor in mice
}

\author{
A. C. Cavanagh, H. Morton, S. Athanasas-Platsis, K. A. Quinn and \\ B. E. Rolfe \\ Department of Surgery, University of Queensland, Royal Brisbane Hospital, Brisbane, \\ Queensland 4029, Australia
}

\begin{abstract}
Summary. Previous studies have indicated that early pregnancy factor (EPF) produced in the pre- and peri-implantation stage of pregnancy appears to consist of inactive components which combine to produce the active species. This is in contrast with EPF produced later in gestation which appears to consist of a single active species. The original studies on ammonium sulphate fractionation of mouse serum and in-vitro culture of mouse ovaries and oviducts have been repeated but tested in the bioassay for EPF, the rosette inhibition test, over an extended range of dilutions. This revealed that the two components in early pregnancy can be understood as EPF and an inhibitor(s). Once this inhibitor is removed, the active fractions in both early and late pregnancy sera exhibit similar behaviour in the above assay. It was shown also that the ovary alone is the source of activity but that this is modulated by an inhibitory substance(s) from the oviduct. Reversed-phase HPLC studies on purified 'early' EPF confirm that active and inhibitory components are present and demonstrate that the active component exhibits an identical elution pattern to 'late' EPF. Thus as pregnancy proceeds, it is not EPF that alters but rather the inhibitor(s), which disappears from the circulation soon after implantation. This substance(s) is under hormonal control, being present during oestrus as well as the early stages of pregnancy; it may be an important biological regulator of EPF. Its action in the rosette inhibition test has profound implications for further study using this bioassay.
\end{abstract}

Keywords: early pregnancy factor, rosette inhibition test; inhibitor; mouse

\section{Introduction}

Since early pregnancy factor (EPF) was first described as a pregnancy-associated substance, present in serum of mice within $6 \mathrm{~h}$ of fertile mating (Morton et al., 1976), many groups have investigated aspects of its biological properties in a wide variety of species. While fewer have attempted to isolate the active agent, several reports on the purification of EPF-active substances have appeared to date. These include preparations from sera of pregnant ewes (Wilson et al., 1983), sheep placentae (Clarke et al., 1987), medium conditioned by oestrous mouse ovaries and oviducts stimulated with prolactin and medium conditioned by mouse embryos (Cavanagh, 1984), medium conditioned by the human choriocarcinoma cell-line BeWo (Cavanagh, 1985), and human pregnancy serum (Mehta et al., 1989). There are insufficient data to speculate about the relationship of these substances to each other and thorough comparison must await the cloning of their respective genes. Nevertheless, we have undertaken the preliminary investigations below to clarify one of the more puzzling aspects of the biochemical properties of EPF described to date.

Several studies have suggested that EPF produced early in pregnancy differs from that produced later in gestation. From investigations in the mouse, Morton et al. (1980) concluded that 
during the pre- and peri-implantation stage, the maternal oviduct and ovary each produce an inactive component, termed EPF-A and EPF-B respectively, which combine to produce EPF. These components have counterparts, produced by fractionation of early pregnancy serum with $40 \%$ saturated ammonium sulphate (Clarke et al., 1980); the supernatant corresponds with EPF-A and the precipitate with EPF-B. More recent experiments in the rabbit have confirmed these results (Suoeka et al., 1989). In contrast, serum obtained later in gestation is not split into two inactive components by $40 \%$ ammonium sulphate (Wilson et al., 1984); a single active species appears to be present, whose source is fetal (Morton et al., 1982; Clarke \& Wilson, 1982; Clarke et al., 1987).

Of the purified substances noted above, the material isolated from medium conditioned by mouse oviducts and ovaries (mEPF) is the only example of the 'early' form of EPF. Consistent with the studies above, it appears to consist of inactive components which in combination are active; the other substances apparently consist of a single active component. Nevertheless, studies in this laboratory on the EPF-active molecule isolated from BeWo-conditioned medium (hEPF), representative of the 'late' form of EPF, indicate that it is closely related to 'early' EPF (mEPF). An immunoabsorbent prepared from anti-mEPF could be used to purify hEPF (Cavanagh, 1985) while antibodies to hEPF, administered to mice on Days 1 and 2 of pregnancy, interrupt gestation in a significant number of cases (Athanasas-Platsis et al., 1989). However, hEPF is considerably more potent (approximate lowest active dose of $\mathrm{hEPF}=50 \mathrm{fg} / \mathrm{ml}, \mathrm{mEPF}=100 \mathrm{pg} / \mathrm{ml}$; Cavanagh 1984, 1985) in the bioassay for EPF, the rosette inhibition test (Morton et al., 1974, 1987; Rolfe et al., 1984). This suggests that an inhibitor(s) might be co-purifying with mEPF. If this is considered in conjunction with the bell-shaped dose-response curve which both molecules exhibit in the bioassay (i.e. high as well as low concentrations give negative results; Cavanagh, 1984, 1985), then it is clear that a re-analysis of previous studies on 'early' EPF might now lead to alternative conclusions.

\section{Materials and Methods}

Animals. Outbred Quackenbush mice, 8-12 weeks old, were used throughout. Stage of the oestrous cycle was determined by vaginal smear (Rugh, 1968). When indicated, ovulation was induced by i.p. injection of 10 i.u. each of PMSG (Folligon: Intervet, Melbourne, Australia) and hCG (Pregnyl: Organon, Morden, UK) given approximately $48 \mathrm{~h}$ apart (Edwards \& Gates, 1959). Females were caged with males overnight and the presence of a vaginal plug the following morning was taken as evidence of mating (Day 1); pregnancy was confirmed by the presence of fertilized eggs/implantation sites in the reproductive tract.

Assay for EPF by rosette inhibition test This assay is dependent on the original finding of Bach \& Antoine (1968) that an immunosuppressive anti-lymphocyte serum (ALS) can inhibit active rosette formation between lymphocytes and heterologous red blood cells. The highest dilution of an ALS to give significant inhibition (rosette inhibition titre: RIT) does not vary with lymphocytes from different donors. A modification of this assay was introduced to detect EPF after it was demonstrated that lymphocytes, preincubated with EPF, give a significantly higher RIT with an ALS than do lymphocytes from the same donor without EPF (Morton et al., 1974, 1976).

The assay was performed with spleen cells from male mice, rabbit anti-mouse lymphocyte serum and human red blood cells (RBC) (Rolfe et al., 1984; Morton et al., 1987), with one batch of ALS used throughout. For each test, $1.8 \times 10^{7}$ freshly isolated spleen cells were incubated at $37^{\circ} \mathrm{C}$ for $0.5 \mathrm{~h}$ with $0.2 \mathrm{ml}$ test sample. After incubation, the cells were washed twice in Hank's balanced salt solution, without calcium or magnesium (HBSS), reconstituted to $1.0 \mathrm{ml}$ in HBSS and used to estimate the RIT of an ALS as described by Rolfe et al. (1984). Positive (purified mouse EPF (see below), $5 \mathrm{ng} / \mathrm{ml}$ in $\mathrm{HBSS} / 0.01 \%(\mathrm{w} / \mathrm{v})$ bovine serum albumin (BSA)] and negative [HBSS $/ 0.01 \%$ BSA] controls were included with each set of tests. RIT is expressed as $\log _{2}$ (reciprocal dilution of ALS $\times 10^{-3}$ ); an RIT $\geq 16$ was positive for EPF and an RIT < 16 was negative for EPF (see Morton et al., 1987).

Before assay, column fractions and azide-containing media were exchanged into PBS $/ 0.01 \%$ BSA on small columns of Sephadex G-25 (PD-10; Pharmacia-LKB, Uppsala, Sweden). Together with serum samples, these were assayed in 10 -fold dilutions prepared in $\mathrm{HBSS} / 0 \cdot 01 \% \mathrm{BSA}$.

Ammonium sulphate fractionation of serum. Serum from 5 mice on Day 2 of pregnancy was pooled and solid ammonium sulphate added to a final saturation of $40 \%$, as described by Clarke et al. (1980). The mixture was kept on ice and stirred gently for $15 \mathrm{~min}$, then centrifuged at $2000 \mathrm{~g}$, for $30 \mathrm{~min}$. The pellet fraction was then reconstituted to the original serum volume with PBS, and this, the supernatant fraction, and a sample of the original serum, dialysed extensively against PBS (Visking cellulose tubing: $M_{\mathrm{r}} 6000-8000$ cut-off). The exper'ment was repeated using pools of 
serum from 5 mice on Day 10 of pregnancy, or in oestrus or dioestrus. Samples of $0 \cdot 1$ ml supernatant or pellet fractions were then combined with $0.1 \mathrm{ml}$ HBSS or $0.1 \mathrm{ml}$ various pellet or supernatant fractions respectively. These were then tested in the rosette inhibition test (dilution $\frac{1}{2}$ ) along with 10 -fold dilutions of the mixtures prepared in HBSS/BSA. The original sera were tested over the same range of dilutions.

The effect on activity of varying the proportions of supernatant and pellet fractions was studied using recombinants of a pool of equal amounts of Day 2 and Day 10 precipitate (Day $2 / 10$ precipitate) and 'oestrous' supernatant. A $0.1 \mathrm{ml}$ sample of a 1 in 10 dilution of Day $2 / 10$ precipitate was combined with $0.1 \mathrm{ml}$ undiluted 'oestrous' supernatant or $0.1 \mathrm{ml}$ undiluted Day $2 / 10$ precipitate was combined with a $0.1 \mathrm{ml}$ sample of various dilutions of 'oestrous' supernatant; the mixtures were then tested in the rosette inhibition test undiluted and in 10 -fold dilutions prepared in HBSS/BSA.

Organ culture. For separate culture, ovaries and oviducts were removed separately from 6 superovulated mice within $20 \mathrm{~h}$ of hCG injection. Two groups of 6 ovaries were cultured in RPMI 1640 with the addition of prolactin and medium conditioned by mouse embryos, while 2 groups of 6 oviducts were each cultured in 5 ml RPMI 1640, as described previously (Cavanagh, 1984). Conditioned medium, alone and in combination, was then tested in the rosette inhibition test over a range of dilutions using the protocol for serum described above.

For joint culture, ovaries and oviducts were removed as a unit from 80 superovulated mice within $20 \mathrm{~h}$ of hCG injection and cultured in RPMI 1640 with prolactin and medium conditioned by mouse embryos, as described previously (Cavanagh, 1984). Conditioned medium was used for purification of $\mathrm{mEPF}$.

Purification of $\boldsymbol{m E P F}$. $m$ EPF was purified from medium conditioned by ovaries and oviducts combined, following the method described by Cavanagh (1984), with the following variations. A second generation immunoabsorbent was used in the initial step, prepared from $300 \mathrm{mg}$ goat $\mathrm{IgG}$ raised against mEPF purified by the original method and coupled to CNBr-activated Sepharose 4B according to the manufacturer's instructions (Pharmacia-LKB). Tween-20 was omitted from the second electrofocusing step and, following the final gel-filtration step, half the preparation was subjected to reversed-phase HPLC (RP-HPLC) under conditions used for purification of hEPF (Cavanagh, 1985). Briefly, trifluoroacetic acid (TFA; Sequenal grade, Pierce Chemical Company, IL, USA) was added to a final concentration of $0.1 \%$, and, after passage through an $0.45 \mu \mathrm{m}$ filter, the sample was applied to a $\mathrm{C}_{3}$ column (Ultrapore RPSC, $4.6 \times 75 \mathrm{~mm}, 5 \mu \mathrm{m}$ particle size, $30 \mathrm{~nm}$ pore size; Beckman Instruments, CA, USA) which had been equilibrated with $0 \cdot 1 \%$ TFA. The column was then eluted with a 2 min linear gradient to $25 \%$ isopropanol (HPLC grade; Mallinckrodt, KE, USA), followed by a 30 min linear gradient to $30 \%$ isopropanol, both containing $0 \cdot 1 \%$ TFA. A flow rate of $1 \mathrm{ml} / \mathrm{min}$ was maintained throughout.

For high-performance gel permeation chromatography (GP-HPLC), samples were applied to a TSK G3000 SW column $(7.5 \times 600 \mathrm{~mm})$ with TSK-GSWP precolumn $(7.5 \times 75 \mathrm{~mm})$ (Pharmacia-LKB), through a $250 \mu \mathrm{l}$ sample loop, using $0 \cdot 1 \mathrm{M}$-sodium phosphate buffer pH 6.8 containing $0 \cdot 1 \%$ sodium dodecyl sulphate (SDS; BDH, Poole, UK) as equilibration and running buffer. A flow rate of $0.5 \mathrm{ml} / \mathrm{min}$ was maintained throughout.

\section{Results}

\section{Ammonium sulphate studies}

The earlier studies of Clarke et al. (1980), which showed that $40 \%$ saturated ammonium sulphate could apparently split EPF activity in early pregnancy serum into two components, were repeated but tested over an extensive range of dilutions, as shown in Fig. 1(a). As was observed originally for Day 2 serum, the $40 \%$ supernatant and precipitate have no activity when tested at a dilution of 1 in 2, but a recombinant does, as does the serum before fractionation. However, when the range of dilutions is extended, it can be seen that the precipitate alone exhibits activity. The supernatant shows no activity at any of the dilutions tested, but is able to reduce the EPF titre of the precipitate, suggesting that an inhibitor(s) is present in this fraction.

In contrast, Fig. 1(b) shows that, when Day 10 serum is fractionated by ammonium sulphate, the $40 \%$ supernatant and precipitate, a recombinant of the two and the original serum, all exhibit essentially the same behaviour in the rosette inhibition test. At low dilutions, positive results are observed although the RIT is low and variable among samples. As the dilution increases, however, superimposable strong positive results are obtained for all samples, suggesting that by this stage of gestation the inhibitor(s) is no longer present and that $40 \%$ ammonium sulphate only partly precipitates EPF.

The relationship of these active and inhibitory fractions during the course of pregnancy was further investigated by mixing Day 10 precipitate with Day 2 supernatant, and vice versa, then testing the combinations as described in Fig. 1. Day 10 precipitate/Day 2 supernatant displays 


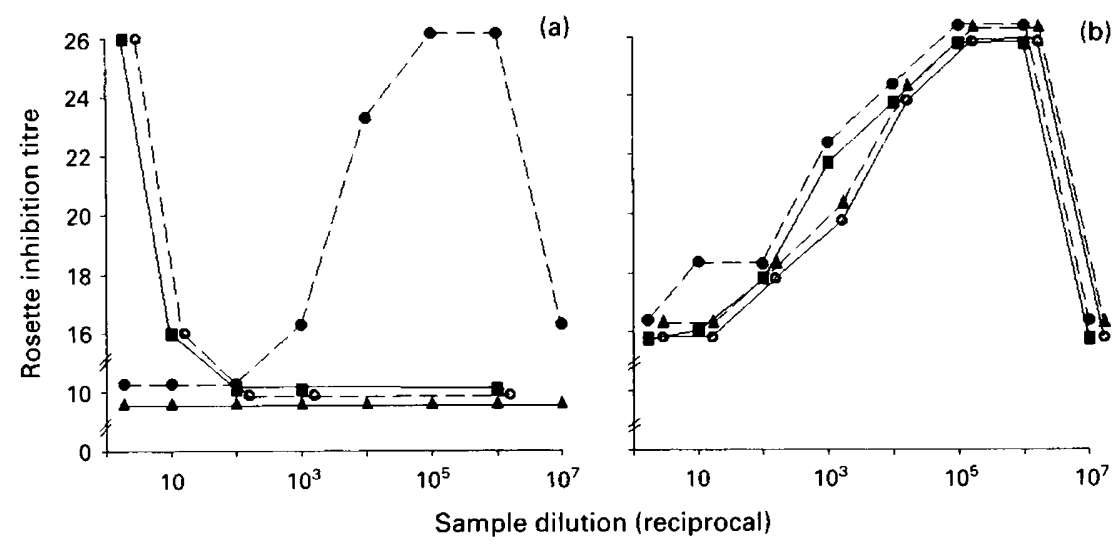

Fig. 1. EPF activity of mouse serum on (a) Day 2 and (b) Day 10 of pregnancy, fractionated by $40 \%$ saturated ammonium sulphate: $\mathbf{D}-\mathbf{\square}$, serum; $\mathrm{O}---\mathrm{O}$, recombinant; $\boldsymbol{\Delta}-\boldsymbol{\Lambda}$, supernatant; - - , precipitate. EPF is expressed as RIT with values of $\geq 16$ indicating a positive result.

essentially the same behaviour as Day 2 serum and recombinant (see Fig. 1a), while Day 2 precipitate/Day 10 supernatant behaves similarly to Day 10 serum and recombinant (see Fig. 1b) (data not shown). The active fractions in both Day 2 and Day 10 serum therefore exhibit similar behaviour in the rosette inhibition test, both when tested alone and in combination with the inhibitory fraction from Day 2 serum, confirming that, as pregnancy proceeds, it is the regulatory rather than the active fraction that alters.

Since the original studies indicated that "EPF-A" was produced by the oviduct during oestrus as well as early pregnancy (Morton et al., 1980), the present studies were extended to include serum from mice in oestrus and dioestrus. As shown in Fig. 2(a) and (b), neither supernatant nor pellet fractions from either source were active when tested over a wide range of dilutions. In Fig. 2(a) it can be seen that 'dioestrous' supernatant has no effect on activity of either Day 2 or Day 10 precipitate (see Fig. la and b, respectively) in contrast with 'oestrous' supernatant (Fig. 2b) which exhibits an inhibitory effect similar to that of Day 2 supernatant (see Fig. 1a). The dose-response of the inhibitory effect of 'oestrous' supernatant on Day $2 / 10$ precipitate is shown in Fig. 2(c). The essentially parallel curves generated either by reducing the proportion of precipitate (active fraction), or progressively reducing the proportion of supernatant (inhibitory fraction), give strong support to the proposition that the results reported in Figs 1 and 2 are indicative of a substance(s) in the supernatant acting as an inhibitor of activity.

\section{Conditioned medium, separate culture of 'oestrous' ovaries and oviducts}

Conditioned medium from separate culture of ovaries and oviducts was tested in the rosette inhibition test alone and in combination, as shown in Fig. 3(a). As was found with the original studies (Morton et al., 1980), when tested at low dilutions, neither medium conditioned by the ovary nor oviduct alone exhibit activity, but a combination of the two does. However, when the range of dilutions is extended, ovary-conditioned medium alone is found to be active. Medium conditioned by the oviduct shows no activity at any dilution tested but is able to reduce considerably the EPF titre of ovary-conditioned medium, suggesting that it is a source of the inhibitor(s) identified above. In Fig. 3(b) the dose-response of this inhibitory effect is shown with parallel curves again being generated, suggesting that mechanisms similar to those seen with serum (Figs 1 and 2) are operating. This is confirmed in Fig. 3(c) in which it can be seen that the inhibitory effect of 'oestrous' supernatant and oviduct-conditioned medium is essentially interchangeable, as is the response of Day 2/10 precipitate and ovary-conditioned medium. 

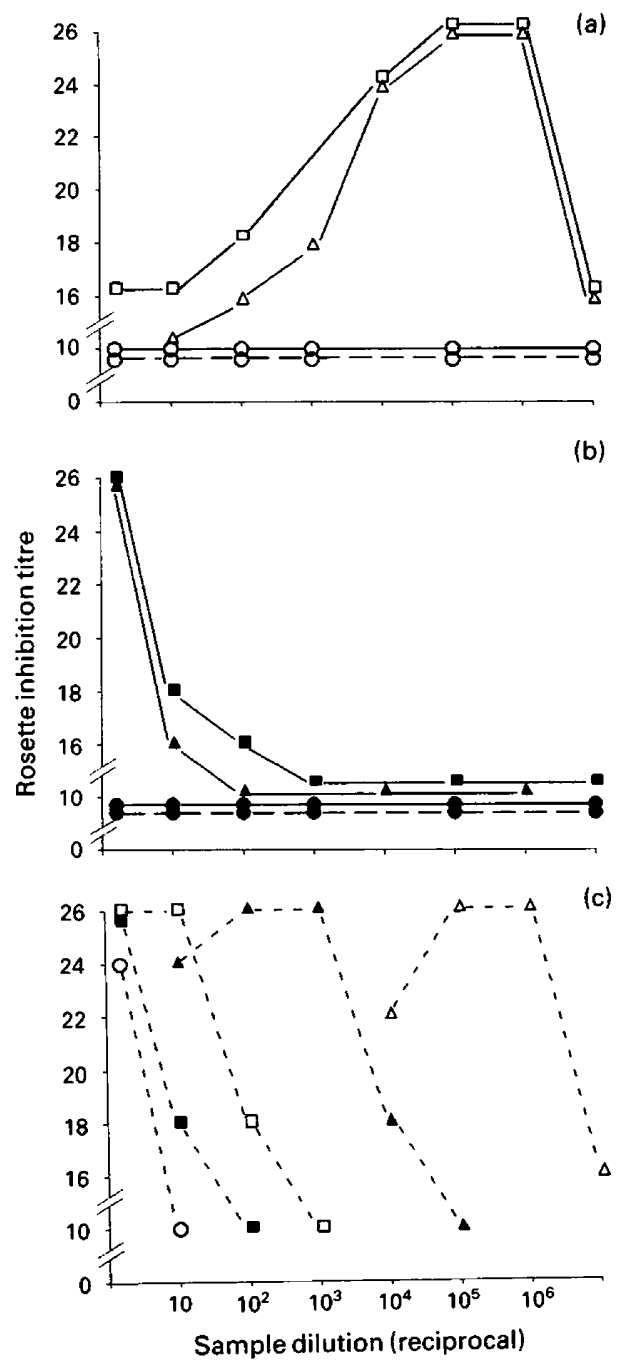

Fig. 2. Effect of (a) dioestrous and (b) oestrous mouse serum, fractionated by $40 \%$ saturated ammonium sulphate, on EPF activity of $40 \%$ saturated ammonium sulphate precipitates of Day 2 and Day 10 pregnant mouse sera combined in equal proportions, and of (c) 'oestrous' supernatant and Day $2 / 10$ precipitate combined in various proportions. (a) $\bigcirc-O$, 'dioestrous' supernatant; $O---O$, 'dioestrous' precipitate; $\triangle \longrightarrow \Delta$, Day 2 precipitate + 'dioestrous' supernatant; $\square-\square$, Day 10 precipitate + 'dioestrous' supernatant. (b) $\square-\emptyset$, 'oestrous' supernatant; $---\mathbf{O}$, 'oestrous' precipitate; $\boldsymbol{\Delta}-\boldsymbol{\Delta}$, Day 2 precipitate + 'oestrous' supernatant; $\mathbf{\square}$, Day 10 precipitate + 'oestrous' supernatant. (c) $\bigcirc \cdots \bigcirc$, Day $2 / 10$ precipitate $10^{-1}+$ 'oestrous' supernatant; $\cdots \mathbf{D}$, Day $2 / 10$ precipitate + 'oestrous' supernatant; $\square \cdots \square$, Day $2 / 10$ precipitate + 'oestrous' supernatant $10^{-1} ; \boldsymbol{\Delta} \cdots \mathbf{\Delta}$, Day $2 / 10$ precipitate + 'oestrous' supernatant $10^{-3} ; \Delta \cdots \Delta$, Day $2 / 10$ precipitate + 'oestrous' supernatant $10^{-5}$. EPF is expressed as RIT with values of $\geq 16$ indicating a positive result.

\section{HPLC studies}

The RP-HPLC profile of mEPF is shown in Fig. 4, while Table 1 shows results of testing, in the rosette inhibition test, fractions, pooled as indicated in Fig. 4, alone and in combination. The most notable features of this experiment are (a) that the elution position of mEPF (pool 2) is virtually identical to hEPF (see Cavanagh, 1985); (b) a substantial increase in EPF titre is observed upon 

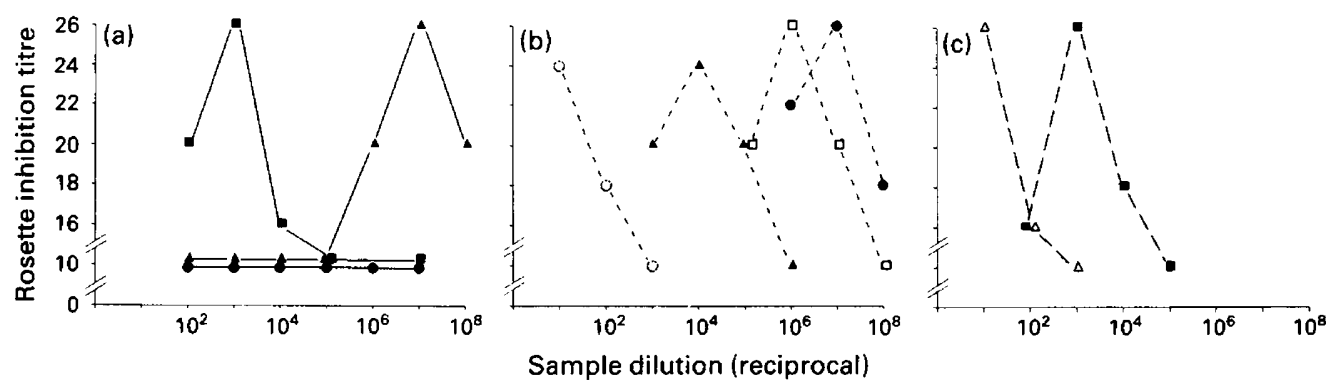

Fig. 3. EPF activity of conditioned medium from separate culture of oestrous mouse oviducts, and ovaries stimulated with prolactin and mouse embryo-conditioned medium, (a) alone and combined in equal proportions, (b) combined in various proportions, and (c) combined with $40 \%$ saturated ammonium sulphate precipitate of Day 2 and 10 pregnant mouse sera and $40 \%$ saturated ammonium sulphate supernatant of oestrous mouse serum respectively. (a)

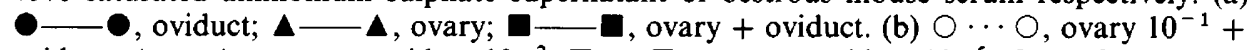
oviduct; $\boldsymbol{\Delta} \cdots \boldsymbol{\Delta}$, ovary + oviduct $10^{-2} ; \square \cdots \square$, ovary + oviduct $10^{-5} ; \bullet \cdots \bullet$, ovary + oviduct $10^{-7}$. (c) $\triangle---\triangle$, oviduct + Day $2 / 10$ precipitate; $\mathbf{-}--\mathbf{0}$, ovary + 'oestrous' supernatant. EPF is expressed as RIT with values of $\geq 16$ indicating a positive result.

RP-HPLC, indicating that an inhibitor(s) may have been removed, and (c) this inhibitory substance is present in pool 5 .

The dose-responses of MEPF before RP-HPLC, the active fraction after RP-HPLC (pool 2, Fig. 4) and a recombinant of this and the inhibitory fraction separated by RP-HPLC (pool 5, Fig. 4) are shown in Fig. 5. As was seen with serum and conditioned medium, the effect of the inhibitor is to induce a substantial shift in the dose-response curve indicating that the isolated substance(s) is acting in a manner similar to the inhibitory fractions of both serum-free conditioned medium (source material) and serum.

Since the apparent components in MEPF, as originally purified, were analysed by GP-HPLC in the presence of SDS (Cavanagh, 1984), this technique was applied to the active and inhibitory fractions produced by RP-HPLC, along with an equivalent amount of the mEPF preparation before RP-HPLC. As shown in Fig. 6, mEPF and the inhibitor(s) co-eluted, even under dissociating conditions. In the original studies, SDS appeared to split EPF into three peptides, none of which had activity in the rosette inhibition test. When equal amounts of all three, but not of any two, were mixed, activity was again observed. However, peaks and combinations were tested at a single dilution. At that stage, no studies had been performed on BeWo conditioned medium and there was no reason to test beyond the range observed with ovary-oviduct conditioned medium. If the range of dilutions had been extended, the studies in Fig. 6 suggest that a positive result would have been obtained within the area of elution but, as originally tested, it was not until sufficient amounts of inhibitor had been added (i.e. all 3 peaks were mixed) that the EPF titre was reduced to the dilution tested. Nevertheless, while the breadth of the peaks in Figs 6(b) and (c) do suggest heterogeneity, they do not reproduce the original studies. Having established conditions for separation of the inhibitor(s) from EPF, it will be possible to determine whether the 3 peaks originally seen form a complex or whether some were chance contaminants, subsequently eliminated by use of a more specific immunoabsorbent.

\section{Discussion}

The studies described above flow from insights into the mechanism of action of EPF in the rosette inhibition test, gained through attempts to isolate the active species. The assay is essentially nonquantitative and the RIT of a sample is not a reliable measure of EPF concentration. The assay can be used in a semi-quantitative manner by testing samples over a range of dilutions and determining 
Table 1. Activity of mEPF before and after RPHPLC

\begin{tabular}{lcc}
\hline & \multicolumn{2}{c}{ EPF† } \\
\cline { 2 - 3 } Sample* & Alone & $\begin{array}{c}\text { Combined } \\
\text { with RP-HPLC } \\
\text { pool 2 }\end{array}$ \\
\hline mEPF, original & $10^{6}$ & NT \\
technique & NA & $10^{9}$ \\
RP-HPLC pool 1 & $10^{9}$ & NT \\
RP-HPLC pool 2 & NA & $10^{9}$ \\
RP-HPLC pool 3 & NA & $10^{9}$ \\
RP-HPLC pool 4 & NA & $10^{6}$ \\
RP-HPLC pool 5 & \\
\hline
\end{tabular}

*EPF-active fractions from the final gel filtration step of the original technique used for preparation of $\mathrm{mEPF}$ were subjected to RP-HPLC and resultant fractions pooled as shown in Fig. 4. Pools were then tested alone and in the combinations shown.

$†$ Results are expressed as the reciprocal of the highest dilution of sample to give activity in the assay. The lowest dilution tested was 1 in $10^{3}$. NA is no activity, NT is not tested.

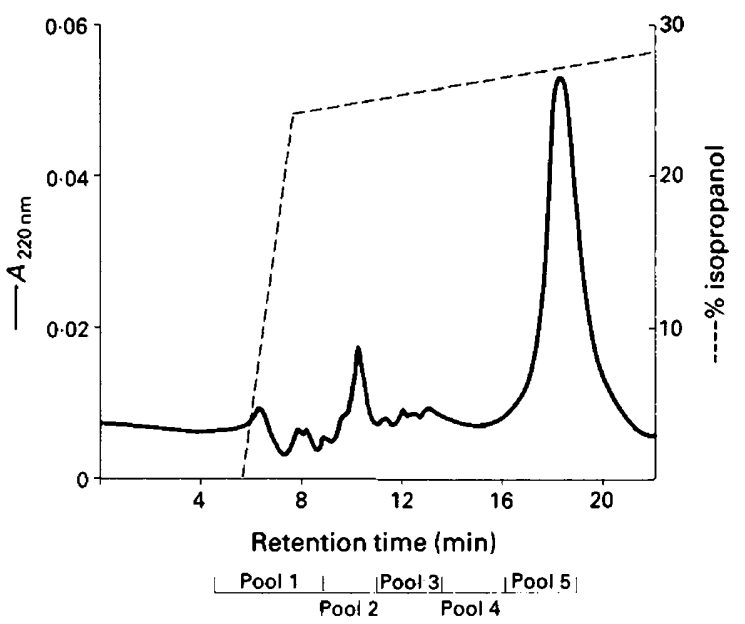

Fig. 4. RP-HPLC profile of mEPF eluted from $C_{3}$ column with isopropanol -- -; optical density monitored continuously at $220 \mathrm{~nm} \_$; fractions pooled as indicated.

a limiting positive dose or EPF titre. This is readily applicable to serum and urine samples since initial studies found that pregnancy serum and urine were reactive in this assay at a dilution of 1 in 2 up to $>1$ in 100. As discussed previously, a more complex bell-shaped dose-response is seen when testing purified or partly purified material, an observation made also by Wilson et al. (1983) and Clarke et al. (1987). While the mechanism of this loss of response at high doses of EPF had not been studied closely, the observations of Rolfe et al. (1988) may provide an explanation. These authors showed that dibutyryl cyclic adenosine mono-phosphate (cAMP) stimulates releases of the genetically restricted suppressor factors which are the active species in the rosette inhibition test. This suggests that EPF acts via a classical receptor pathway mediated by adenylate cyclase in which cAMP acts as a second messenger (Robinson et al. (1971). The adenylate cyclase system displays hormone-specific desensitization (uncoupling of receptor from adenylate cyclase) which can occur 


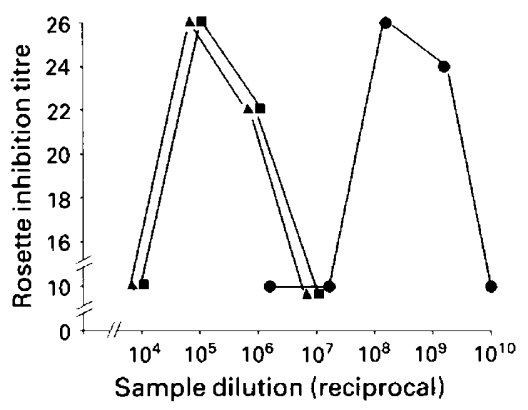

Fig. 5. EPF activity of mEPF ( $\boldsymbol{\Lambda}-\mathbf{\Lambda})$ before RP-HPLC; $-\boldsymbol{-}$, active fraction after RP-HPLC (pool 2, Fig. 4); $\square-\mathbf{\square}$, recombinant of active and inhibitory fractions after RP-HPLC (pools 2 and 5 respectively, Fig. 4). EPF is expressed as RIT with values of $\geq 16$ indicating a positive result.

(a)

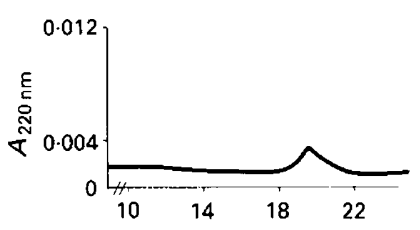

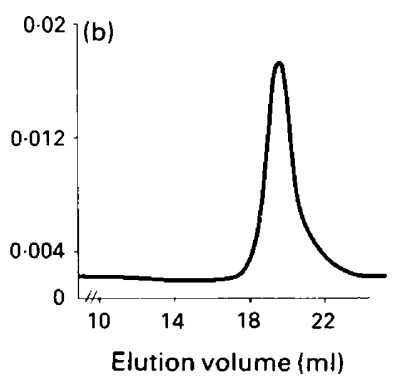

Fig. 6. GP-HPLC profile of mEPF-RP-HPLC fractions (see Fig. 4): (a) pool 2; (b) pool 5; (c) $\mathrm{mEPF}$ before RP-HPLC fractionation; optical density monitored continuously at $220 \mathrm{~nm}$.

very rapidly at saturating doses of hormone (see for example Su et al., 1980) and results in a bellshaped dose-response. Clarke et al. (1987) have commented on other qualitative aspects of the assay, noting that activity may be modulated by the presence of other factors. This can be seen above when comparing the wide range of response of serum (Fig. 1) with the increasingly restricted range of response of serum-free conditioned medium (Fig. 3) and highly purified material (Fig. 5). Finally, as Fig. 1 graphically illustrates, activity can alter dramatically once complex biological mixtures are biochemically manipulated. All of these observations are indicative of the nature of the bioassay, a complex cascade of events involving specifically activated spleen cells. EPF initiates the cascade but different substances may intervene at different points with varied effects on the final outcome; effector molecules downstream of the inducing stimulus may be used to short-circuit the cascade. While the studies above focus on one modifier which acts very early in the cascade, they should be understood in this wider context.

The identification of this modifier and its effects has profound implications for future study using the rosette inhibition test. The difference in titre of 4-5 orders of magnitude observed in samples with and without inhibitor is of critical importance, for example, in valid comparison of serum from males and females. It is even more relevant in the daunting process of isolating such highly active molecules from complex biological mixtures. The incorrect assumption by Mehta et al. (1989), that specific activity is constant, renders meaningless the calculations used by this group in assessing source material and fractionation procedures. Even more alarming is the use by Bose et al. (1989) of an individual RIT to assess isolation of an EPF-active substance from medium conditioned by human embryos. A brief reflection on the effect on activity of a simple biochemical procedure, such as that demonstrated in Fig. 1, suggests that the evaluation procedures used 
by Bose et al. (1989) would almost certainly miss the most active fractions. It is probable that the activity shown by the isolated $M_{\mathrm{r}} 14000$ substance reflects the presence of a very minor contaminant.

Nevertheless, when used with care, the rosette inhibition test is invaluable in resolving at least some of the complexity associated with EPF. In the studies above, it could determine that the differences previously observed between the so-called 'early' and 'late' forms of EPF are the result of modulation of the activity of EPF in the rosette inhibition test by a substance(s) present during oestrus and the early stages of gestation. The substance(s) has been termed an inhibitor because it substantially reduces the titre of EPF in the above assay. The two components thought to be present early in pregnancy can be understood therefore as EPF and inhibitor(s); EPF-B becomes EPF, EPF-A the inhibitor(s). Thus as pregnancy proceeds, it is not EPF that alters but rather the inhibitor which disappears from the circulation soon after implantation (see also Fig. 3, Morton $e t$ al., 1983; Fig. 2, Morton, 1984).

The proposition that 'EPF-A' is a regulatory rather than active component is supported by the HPLC studies above. As shown in Figs 4 and 5 and Table 1, when mEPF ('early') is subjected to RP-HPLC, a substantial increase in EPF titre occurs. mEPF now appears to exhibit similar potency in the rosette inhibition test and displays a similar elution profile to hEPF ('late'). However, a fraction separated from mEPF by RP-HPLC (pool 5; see Fig. 4), while exhibiting no activity itself, is able to reduce this titre to the level observed before RP-HPLC. Although preliminary, these studies reveal that, when analysed by one of the highest resolution techniques currently available, mEPF ('early') and hEPF ('late') display significant similarities. Furthermore, by demonstrating that the regulatory and active components can be separated, they make it possible to study the biological significance of the regulatory substance(s).

The inhibitor(s) modifies the action of EPF on lymphocytes. In its presence, higher concentrations of EPF are required to stimulate the suppressor release by which the action of EPF in the rosette inhibition test and the delayed-type hypersensitivity reaction is mediated (Rolfe et al., 1988, 1989). The range of concentrations over which EPF is able to affect lymphocytes is thus extended, suggesting a possible biological function for the regulator. That this substance does have a role is reinforced by the observation that production of the inhibitor is under hormonal control, being produced by the oviduct during oestrus and early pregnancy (Morton et al., 1980; see also Morton (1984) for an illustration of the effect of the oestrous cycle on EPF-A in serum). A larger $M_{\mathrm{r}}$ binding protein (or group of proteins; $\left.M_{\mathrm{r}}>100000\right)$ also associates with EPF in serum during the pre- and periimplantation period. It appears to act as a storage protein for EPF and has been observed in mice (Clarke et al., 1978), sheep (Clarke et al., 1980), pigs (Morton et al., 1983) and humans (Rolfe et al., 1983). EPF can be separated from the protein(s) by anion exchange chromatography (Clarke et al., 1980 ) but its activity is not altered during this process. These results are in contrast with the properties of the inhibitory substance discussed above but, when taken together, suggest a mechanism of both coarse (storage/protein carrier) and fine (inhibitory protein carrier) regulation of EPF activity. An analogous situation has been described for control of insulin-like growth factors (IGFs). Large $\left(M_{\mathrm{r}} 150000\right)$ and smaller $\left(M_{\mathrm{r}} 35000\right)$ binding proteins have been identified, with the former binding the majority of IGFs in serum and performing a storage function, while the latter is itself subject to hormonal control, which, in turn, closely regulates IGF function (Hall et al., 1988).

It may be that the co-purification of the inhibitory substance(s) with EPF is fortuitous and its effect in the rosette inhibition test is unrelated to EPF. Alternatively it may be an indication that, like many hormones and growth regulators, EPF activity in vitro and in vivo may be modulated by a complex balance of inhibitors and activators. Now that at least one source of a regulator is known and a technique established to separate it from EPF, more definitive studies on the relationship of the substances can begin.

We thank Ms J. Corcoran for typing the manuscript and Ms B. Menzies and Dr G. Pollock for graphics. This work was supported by the National Health and Medical Research Council. 


\section{References}

Athanasas-Platsis, S., Quinn, K.A., Wong, T-Y., Rolfe, B.E., Cavanagh, A.C. \& Morton, H. (1989) Passive immunisation of pregnant mice against early pregnancy factor (EPF) causes loss of embryonic viability. J. Reprod. Fert. 87, 495-502.

Bach, J.F. \& Antoine, B. (1968) In vitro detection of immunosuppressive activity of anti-lymphocyte sera. Nature, Lond. 217, 658-659.

Bose, R., Cheng, H., Sabbadini, E., McCoshen, J., Madadevan, M.M. and Fleetham, J. (1989) Purified human early pregnancy factor from preimplantation embryos possesses immunosuppressive properties. Am. J. Obstet. Gynecol. 160, 954-960.

Cavanagh, A.C. (1984) The production in vitro of mouse early pregnancy factor and purification to homogeneity. J. Reprod. Fert. 71, 581-592.

Cavanagh, A.C. (1985) Purification and partial characterisation of EPF. In Early Pregnancy Factors, pp. 179-189. Eds F. Ellendorff \& E. Koch. Perinatology Press, New York.

Clarke, F.M. \& Wilson, S. (1982) Biochemistry of early pregnancy factor. In Pregnancy Proteins, pp. 407412. Eds J. D. Grudzinskas, B. Teisner \& M. Seppala. Academic Press, Sydney.

Clarke, F.M., Wilson, S., McCarthy, R., Perkins, T. \& Orozco, C. (1987) Early pregnancy factor: large scale isolation of rosette inhibition test-active polypeptides from ovine placental extracts. $J$. Reprod. Immunol. 10, $133-156$.

Clarke, F.M., Morton, H. \& Clunie, G.J.A. (1978) Detection and separation of two serum factors responsible for depression of lymphocyte activity in pregnancy. Clin. exp. Immunol. 32, 318-323.

Clarke, F.M., Morton, H., Rolfe, B.E. \& Clunie, G.J.A. (1980) Partial characterisation of early pregnancy factor in the sheep. J. Reprod. Immunol. 2, 151-162

Edwards, R.G. \& Gates, A.H. (1959) Timing of the stages of the maturation divisions, ovulation, fertilisation and the first cleavage of adult mice treated with gonadotrophins. J. Endocr. 18, 292-304.

Hall, K., Lundin, G. \& Povoa, G. (1988) Serum levels of the low molecular weight form of insulin-like growth factor binding protein in healthy subjects and patients with growth hormone deficiency, acromegaly and anorexia nervosa. Acta endocr., Copenh. 118, 321-326.

Mehta, A.R., Eessalu, T.E. \& Aggarwal, B.B. (1989) Purification and characterization of early pregnancy factor from human pregnancy serum. J. biol. Chem. 264, 2266-2271.

Morton, H. (1984) Early pregnancy factor (EPF): a link between fertilisation and immunomodulation. Aust. J. biol. Sci. 37, 393-407.

Morton, H., Hegh, V. \& Clunie, G.J.A. (1974) Immunosuppression detected in pregnant mice by rosette inhibition test. Nature, Lond. 249, 459-560.

Morton, H., Hegh, V. \& Clunie, G.J.A. (1976) Studies of the rosette inhibition test in pregnant mice: evidence of immunosuppression? Proc. R. Soc. B 193, 413-419.
Morton, H., Rolfe, B.E., McNeill, L., Clarke, P., Clarke, F.M. \& Clunie, G.J.A. (1980) Early pregnancy factor: tissues involved in its production in the mouse. $J$. Reprod. Immunol. 2, 73-80.

Morton, H., Rolfe, B. \& Cavanagh, A. (1982) Early pregnancy factor: biology and clinical significance. In Pregnancy Proteins, pp. 391-405. Eds J. G. Grudzinskas, B. Teisner \& M. Seppala. Academic Press, Sydney.

Morton, H., Morton, D.J. \& Ellendorff, F. (1983) The appearance and characteristics of early pregnancy factor in the pig. J. Reprod. Fert. 68, 437-446.

Morton, H., Rolfe, B.E. \& Cavanagh, A.C. (1987) Ovum factor and early pregnancy factor. In Current Topics in Developmental Biology, Vol. 23, 73-92. Eds A. McLaren \& G. Siracusa. Academic Press, San Diego.

Robinson, G.A., Butcher, R.W. \& Sutherland, E.W. (1971) Cyclic AMP, pp. 22-24. Academic Press, New York.

Rolfe, B.E., Morton, H. \& Clarke, F.M. (1983) Early pregnancy factor is an immunosuppressive contaminant of commercial preparations of human chorionic gonadotrophin. Clin. exp. Immunol. 51, 45-52.

Rolfe, B.E., Cavanagh, A.C., Forde, C., Bastin, F., Chen, C. \& Morton, H. (1984) Modified rosette inhibition test using mouse lymphocytes for detection of early pregnancy factor in human serum. $J$. immunol. Methods 70, 1-11.

Rolfe, B.E., Cavanagh, A.C., Quinn, K.A. \& Morton, H. (1988) Identification of two suppressor factors induced by early pregnancy factor. Clin. exp. Immunol. 73, 219-225.

Rolfe, B.E., Quinn, K., Athanasas, S., Cavanagh, A.C. \& Morton, H. (1989) Genetically restricted effector molecules released by human lymphocytes in response to early pregnancy factor. Immunol. Cell Biol. 67, 205208.

Rugh, R. (1968) The Mouse: Its Reproduction and Development, pp. 38-39. Burgess Publishing Company, Minneapolis.

Su, Y-F., Harden, T.K. \& Perkins, J.P. (1980) Catecholamine - specific desensitization of adenylate cyclase. Evidence for a multistep process. J. biol. Chem. 255, 7410-7419.

Sueoka, K., Dharmarajan, A.M., Miyazaki, T., Atlas, S.J. \& Wallach, E.E. (1989) In-vivo and in-vitro determination of components of rabbit early pregnancy factors. J. Reprod. Fert. 87, 47-53.

Wilson, S., McCarthy, R. \& Clarke, F.M. (1983) In search of early pregnancy factor: isolation of active polypeptides from pregnant ewes sera. J. Reprod. Immunol. 5, 275-286.

Wilson, S., McCarthy, R. \& Clarke, F. (1984) In search of early pregnancy factor: characterization of active polypeptides isolated from pregnant ewes' sera. $J$. Reprod. Immunol. 6, 253-260.

Received 9 May 1990 